\title{
Three cases of Actinobacillus suis in eastern North Carolina
}

\author{
Emily Mahan-Riggs, DVM
}

\begin{abstract}
Summary
This case study describes sudden deaths of pigs in a North Carolina production system at three different finishing facilities between June 2019 and February 2020. The cases involved 3- to 6-month-old pigs of the same genetics but from different sow farm flows. Pigs at all three sites had gross lesions that included firm, deep red to purple lungs. Based on laboratory results from tissue samples, all cases were diagnosed with Actinobacillus suis. Treatments and the significance of this disease in swine are discussed.
\end{abstract}

Keywords: swine, Actinobacillus suis, bronchopneumonia

Received: November 23, 2020

Accepted: May 12, 2021

\section{Resumen - Tres casos de Actinobacillus suis en el este de Carolina del Norte}

Este estudio de caso describe muertes repentinas de cerdos en un sistema de producción de Carolina del Norte en tres diferentes instalaciones de engorda entre junio de 2019 y febrero de 2020. Los casos incluyeron cerdos de 3 a 6 meses de la misma genética, pero de diferentes flujos de granjas de hembras. Los cerdos en los tres sitios tenían lesiones macroscópicas que incluían pulmones firmes, de color rojo intenso a púrpura. Según los resultados de laboratorio de las muestras de tejido, todos los casos fueron diagnosticados con Actinobacillus suis. En este estudio se discuten los tratamientos y la importancia de esta enfermedad en cerdos.

\section{Résumé - Trois cas d'infection par Acti- nobacillus suis dans l'est de la Caroline du Nord}

Cette étude de cas décrit des morts subites de porcs dans un système de production en Caroline du Nord dans trois installations d'engraissement différentes entre juin 2019 et février 2020. Les cas concernaient des porcs âgés de 3 à 6 mois de la même génétique mais provenant de différents flux d'élevage de truies. Les porcs des trois sites présentaient des lésions macroscopiques qui incluaient des poumons fermes, rouge foncé à violet. Sur la base des résultats de laboratoire d'échantillons de tissus, tous les cas ont été diagnostiqués avec la présence d'Actinobacillus suis. Les traitements et l'importance de cette maladie chez le porc sont discutés.
$\mathrm{F}$ irst reported in 1962, Actinobacillus suis infection was a neonatal or recently weaned pig disease in highhealth or start-up herds. ${ }^{1-10}$ However, disease incidence increased in the 1980s and early 1990s with the implementation of management practices that decreased pig exposure to endemic pathogens..$^{2,4,11}$ Now, the disease is increasingly reported in grow-finish pigs from commercial farms with a normal parity structure..$^{1,3,5,9}$ Due to its similarities to Actinobacillus pleuropneumoniae (APP) infection, it may be underdiagnosed in the field; therefore, prevalence is thought to be underreported. ${ }^{1-3,6,7,9-11}$ From 2018 to 2020, 7 cases of $A$ suis infection were diagnosed in a large commercial hog production system in eastern North Carolina. Three of these cases are described and discussed herein.

\section{Case description}

\section{Case 1}

The first case occurred in June 2019 at a 4-barn finishing site that shared a premises, driveway, and management with another 2-barn finishing site. The barns were partially slatted and naturally ventilated, 1224-head capacity buildings (4896 total head) with solid block penning. The barn placement populations were 1305 pigs in barn 1, 1326 in barn 2, 1313 in barn 3, and 1299 in barn 4 with a total of 5243 pigs at the site. The pigs at this site were from a porcine reproductive and respiratory syndrome (PRRS) stable and PRRS modified-live virus (MLV) vaccinated flow. Three days prior to the veterinarian's visit, pigs were observed to be in good health. However, pigs began coughing, losing weight, and developed watery diarrhea. These symptoms did not affect all the pigs at the same time, but there were at least $5 \%$ to $10 \%$ of them losing weight or with diarrhea, which fit the criteria for treatment according to company protocol. The pigs were prescribed a 3-day course of water-soluble gentamicin to combat the diarrhea. On the day prior to the veterinarian's visit and sample collection, 5 pigs had died. In barn 2, the weekly total mortality was 17 . In that barn, most of the mortalities occurred in the end pen, which had the feed drop motor. Due to the association with the feed drop motor, stray voltage had already been checked and ruled out as the cause of death. At the time of the veterinarian's visit, the pigs were 21 to 24 weeks of age and had been at the finisher site for 11 to 14 weeks. During the veterinarian's walk-through, one pig was found dead in this pen with red froth coming from the

Smithfield Hog Production, Tarboro, North Carolina.

Corresponding author: Dr Emily Mahan-Riggs, 1604 West St. James Street, Tarboro, NC 27886; Tel: 910-289-1569; Email: emahanriggs@ smithfield.com.

This article is available online at http://www.aasv.org/shap.html.

Mahan-Riggs E. Three cases of Actinobacillus suis in eastern North Carolina. J Swine Health Prod. 2022;30(1):24-30. 
nose. Samples of tonsil, lung, heart, multiple lymph nodes, liver, spleen, kidney, intestine, and colon were taken within an hour of death. During the necropsy, red froth was found in the trachea, and the lungs were deep red to purple (Figure 1). Normal lung tissue was difficult to find. The spleen and lymph nodes were enlarged as well. While it is best practice to sample tissues from at least two pigs, only one pig was found dead during the visit. No additional pigs were euthanized. Oral fluids from pigs in all 4 barns (four tubes) were also collected. All samples were sent to the Iowa State University Veterinary Diagnostic Laboratory (ISU VDL). Histopathology, culture, and polymerase chain reactions (PCRs) were completed on the tissues for PRRS, influenza A virus (IAV), and Mycoplasma hyopneumoniae, and PCR was completed on oral fluids for IAV.

The histopathology showed signs of acute, severe fibrinopurulent bronchopneumonia, which is highly suggestive of an Actinobacillus infection. As is typically observed in an Actinobacillus infection, the alveoli were filled with large numbers of neutrophils, rare hemorrhage, clusters of basophilic bacteria, and abundant proteinaceous fluid (Figure 2). Oat cells (white blood cells, in this case neutrophils, with streaming chromatin) were also observed (Figure 3). In addition, fibrin and neutrophils markedly expanded the interlobular septa and lymphatics. Differentiation between APP and $A$ suis, however, is difficult based on lesions alone, so a bacterial culture was requested. Bacterial culture of the lung tissue confirmed an $A$ suis infection. All PCR testing for the other previously mentioned pathogens were negative. Streptococcus suis was also cultured from the lung and Salmonella typhimurium was cultured from the intestine. The $A$ suis that was cultured was susceptible to ampicillin, ceftiofur, enrofloxacin, florfenicol, gentamicin, spectinomycin, tiamulin, tilmicosin, and trimethoprim/ sulfamethoxazole.

The treatments for the pigs in these barns were determined based on the amount of time prior to selling them to market. Barn 1 was the oldest and least affected, so it did not receive any treatment. However, 14 pigs in that barn died in the last 3 weeks prior to marketing with a total mortality of 51 (3.91\%). Barn 2 was the most affected, but pigs were to be sold within 3 weeks. Ceftiofur hydrochloride (Excenel RTU EZ; Zoetis) was given to pigs in the most affected pens in this barn

Figure 1: Affected pig lung. Lung is not collapsed, dark red to purple, moderately firm, with multifocal moderate fibrin on the pleural surface and multifocal hemorrhages on the pleura.

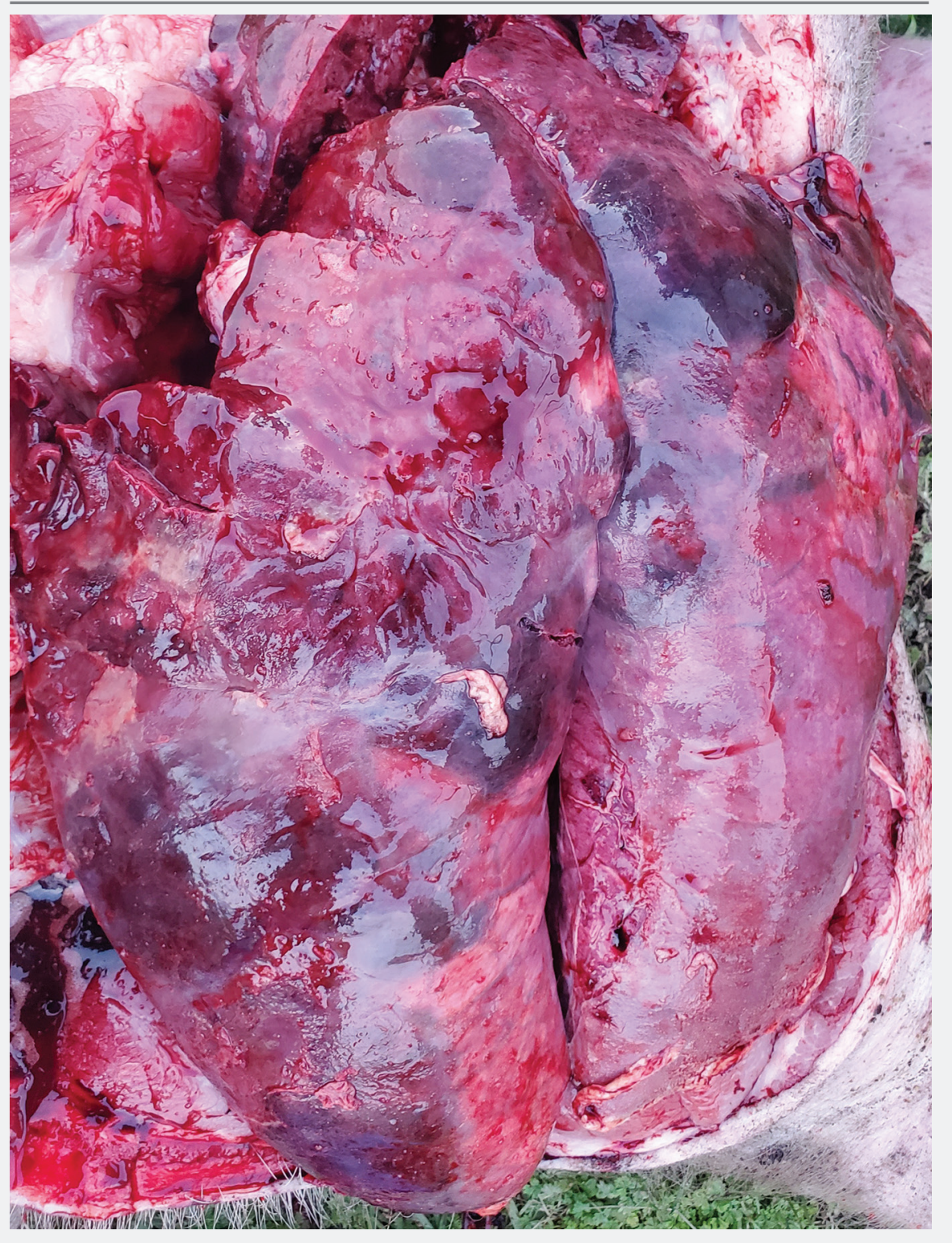

(8 $\mathrm{mL}$ to 122 pigs and $5 \mathrm{~mL}$ to 52 pigs) for 3 consecutive days according to the label. There were 25 more pigs that died in the remaining weeks in barn 2 , and the total mortality was 74 (5.58\%). According to the barn records, no medications were given to barn 3 even though there were 6 weeks left in that barn prior to marketing. During those 6 weeks, 21 more pigs died for a total of 73 (5.56\%). Barn 4 had the youngest pigs on the site with 8 weeks left in the barn. Tiamulin (Denagard; Elanco) was administered at the respiratory level in this barn, and only 22 more pigs died. The total mortality for this barn was 54 (4.16\%). The mean mortality across all 4 barns during this case was $4.81 \%$. Compared to the annual mean mortality in 2018 (4.99\%) and 2019 (5.55\%), mortalities were $0.18 \%$ and $0.74 \%$ less than the historical mean mortality, respectively, for this site during this case (Table 1).

Clinical presentation of $A$ suis and APP infection in pigs are very similar. However, APP infected pigs usually recover once treated, but the probability of relapse is high due to carrier pigs remaining in the population. ${ }^{7}$ Due to the inability to discern between the two Actinobacillus species and the possibility of having new cases, the production personnel were advised to quickly sell the healthy pigs at this site to try to minimize the site's mortality. The 
Figure 2: Serofibrinosuppurative bronchopneumonia with serofibrinous effusion, dense aggregates of neutrophils, and congestion of alveolar capillaries with multifocal hemorrhage. Photo and description courtesy of Dr Greg Stevenson. (Written communication with Greg Stevenson, DVM, PhD, lowa State University Veterinary Diagnostic Laboratory, July 5, 2019.)

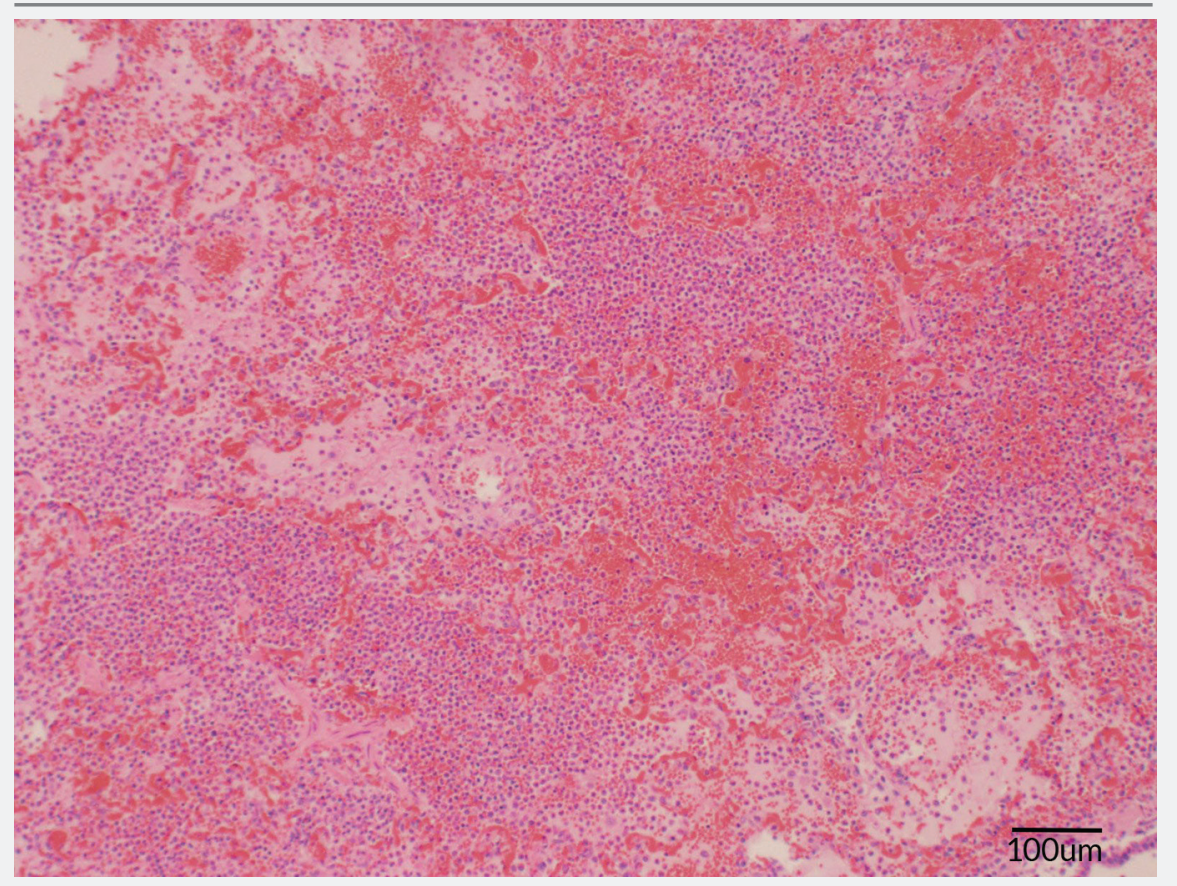

Figure 3: Focal Actinobacillus suis fibrinonecrotic pneumonia. A colony of A suis bacteria (white arrow) is surrounded by infiltrating leukocytes; many are degenerate and streaming "oat cells" (black arrows) due to secreted bacterial leukotoxins. Photo and description courtesy of Dr Greg Stevenson. (Written communication with Greg Stevenson, DVM, PhD, Iowa State University Veterinary Diagnostic Laboratory, January 22, 2021)

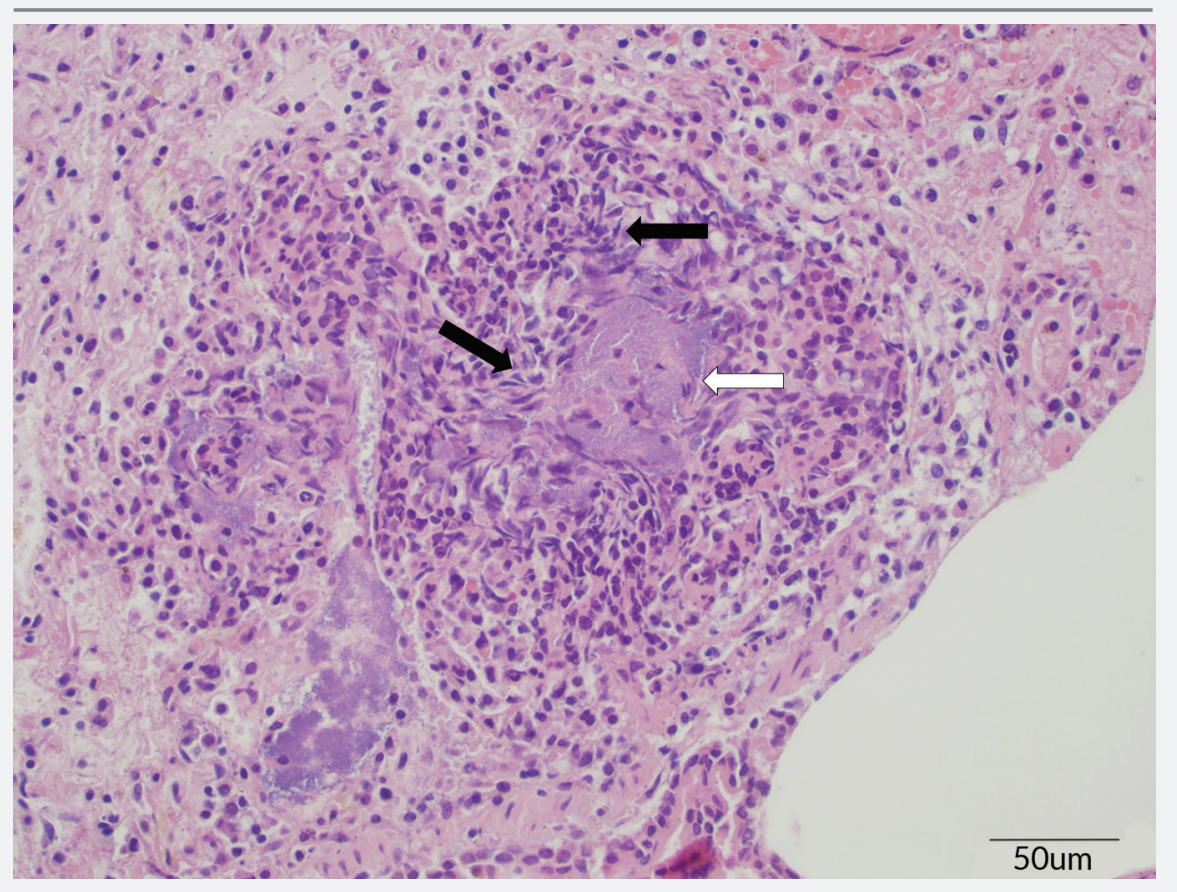

first-in-last-out (FILO) age is a production metric that tracks the number of days between when the first pig arrived at the site and when the last pig was removed from the site. The FILO age for this site during this case was 136.5 days. The FILO age for this flow of pigs was 129.83 days in 2018 and 134.61 days in 2019. The differences between the FILO age in this case and the historical means were 1.89 and 6.67 more days, respectively (Table 1). The older FILO age in this case was likely due to these pigs being placed into the finisher earlier than normal.

\section{Case 2}

The next case occurred the beginning of July 2019. The finishing facility had three, 1160-head capacity barns (3480 total capacity) that were naturally ventilated and fully slatted with metal penning. The barn placement populations were 1225 in barn 1, 1221 in barn 2, and 1247 in barn 3 for a total of 3693 pigs on the site. The flow of pigs at this site was from a previously PRRS-naïve multiplier that broke with PRRS the beginning of April 2019. The timing of the PRRS break on the sow farm meant that the pigs at this finisher were still from a highhealth sow farm at weaning. This highhealth status is often associated with high morbidity and mortality with an $A$ suis infection due to the lack of immunity. ${ }^{2,10}$ The finishing farm staff noticed an increase in mortality 2 weeks before the veterinarian was called to the farm. At that time, the farm administered tiamulin (Denagard; Elanco) at the respiratory level, and the pigs seemed to recover. Then, another increase in mortality started the day before samples were taken. At the time of the veterinarian's visit, the pigs were 21 weeks of age and had been in the finisher for 11 weeks. A hacking cough affected at least $75 \%$ of the pigs in the barns; clear nasal discharge was also observed in these pigs. Those that died were seemingly normal, healthy pigs that died suddenly with bloody discharge from the nose.

Tissues including tonsil, lung, heart, multiple lymph nodes, liver, spleen, kidney, intestine, and colon were taken from 2 pigs soon after death. No oral fluids were collected. During the necropsies, blood was found in the trachea and lungs of both pigs. An enlarged spleen and multiple enlarged lymph nodes were also observed. Samples were sent to the ISU VDL where histopathology, culture, and PRRS and IAV PCRs were completed. Similar to Case 1, the histopathology showed lesions highly suggestive of an 


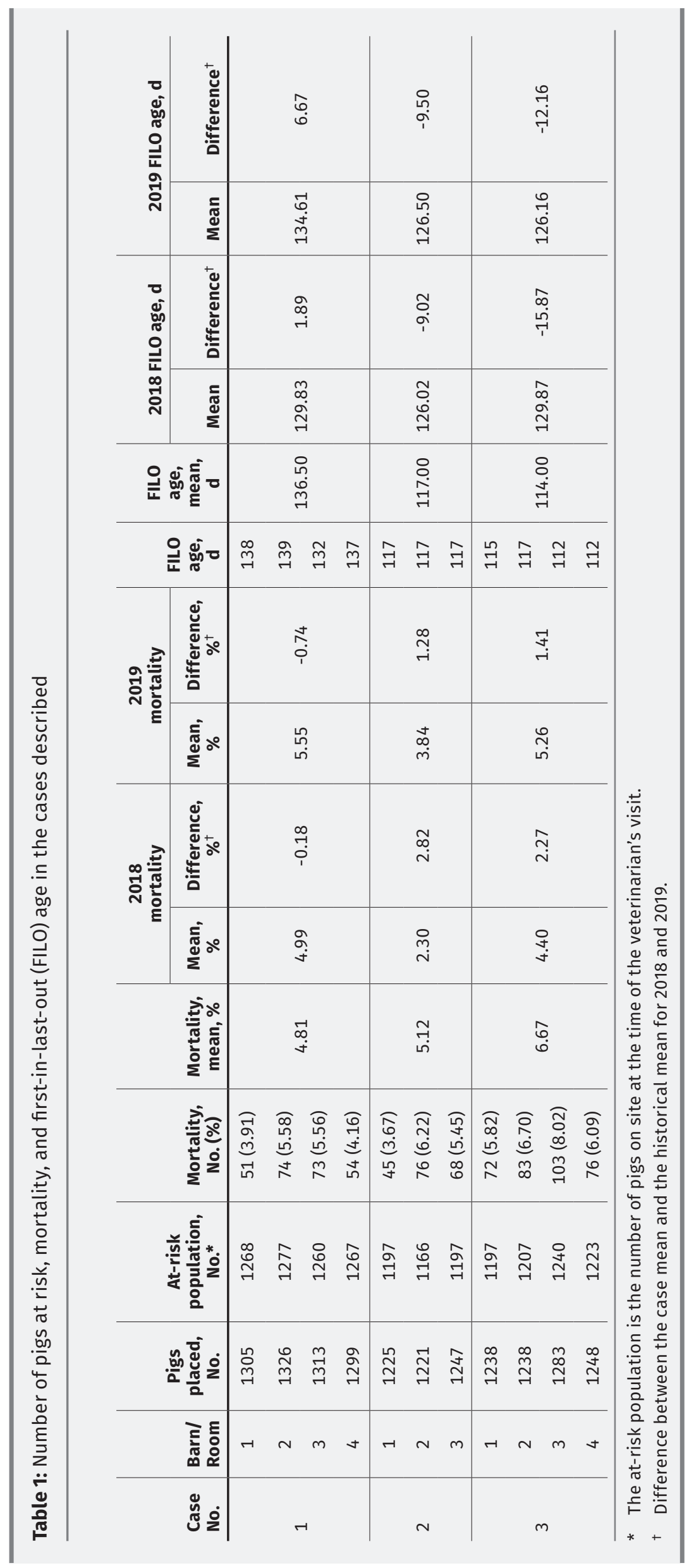

Actinobacillus infection. The blood vessel endothelium was swollen, and serous or serofibrinous fluid that contained occasional aggregates of bacteria expanded the adventitia. Interlobular septa and alveoli were distended by serous or serofibrinous effusion. Most alveoli were filled with dense aggregates of neutrophils and fewer macrophages. There were lesions of acute serofibrinosuppurative bronchopneumonia with intralesional short rod-shaped bacteria (Figures 4 and 5). Acute necrotizing bronchitis and bronchointerstitial pneumonia were also observed. Actinobacillus suis was cultured from the lung, and influenza A(H1N2) virus was identified by PCR. The PRRS PCR was negative, and $S$ suis was also cultured. The $A$ suis that was cultured was susceptible to ampicillin, ceftiofur, enrofloxacin, florfenicol, gentamicin, neomycin, sulfadimethoxine, tiamulin, tilmicosin, and trimethoprim/ sulfamethoxazole.

All 3 barns at this site were treated by administering tiamulin (Denagard) at the respiratory level and injecting the pigs in the most affected pens. Ninetyfive pigs in barn 1, 67 pigs in barn 2, and 36 pigs in barn 3 were injected with 5 $\mathrm{mL}$ of ceftiofur hydrochloride (Excenel RTU EZ) for 3 consecutive days according to the product label. This seemed to halt the high mortality that was seen the week of the veterinarian's visit. Only 7 pigs died in the remaining six weeks in barn 1 with a total mortality of 45 (3.67\%). Barn 2 had 14 more dead pigs after the A suis diagnosis for a total mortality of $76(6.22 \%)$. There were 10 more pigs that died in the last 6 weeks in barn 3 with a total mortality of 68 (5.45\%). The mean mortality across all barns was $5.12 \%$. This is an increase of $2.82 \%$ from the 2018 annual mean mortality $(2.30 \%)$ and an increase of $1.28 \%$ from the 2019 annual mean mortality (3.84\%) for this flow (Table 1). According to Dufresne, ${ }^{3}$ finishing mortality can increase by $1 \%$ to $2 \%$ in a barn of pigs with an $A$ suis diagnosis as is seen in this case.

The marketing of these pigs was handled similarly to Case 1 to try to limit excessive mortality. Production personnel worked diligently to quickly sell the healthy pigs. The FILO age for this site during this case was 117 days. The FILO age of this flow of pigs was 126.02 days in 2018 and 126.5 days in 2019. The differences between this case and the historical means were 9.02 and 9.5 fewer days, respectively (Table 1). 
Figure 4: The epithelium of the bronchiole is thin, irregular, and attenuated, typical of influenza. Blood vessels are congested. The adventitia of the artery in the lower left is distended by serous effusion (edema fluid). Some alveoli contain dense aggregates of bacteria. Photo and description courtesy of $\mathrm{Dr}$ Greg Stevenson. (Written communication with Greg Stevenson, DVM, PhD, lowa State University Veterinary Diagnostic Laboratory, July 5, 2019.)

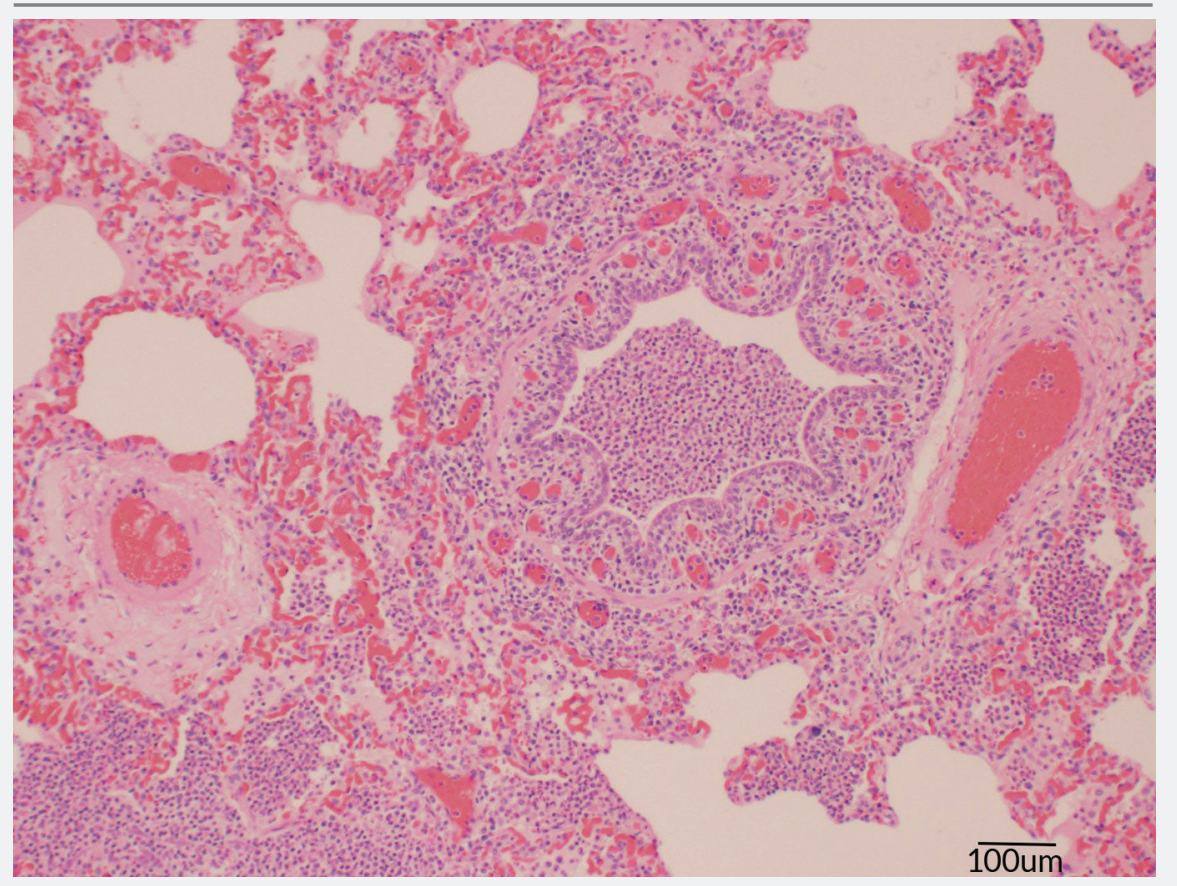

Figure 5: Artery (lower right) has swollen endothelium, and adventitia is distended by edema fluid (between black arrows). Short rod-shaped bacteria are in a vacuole amid the edema fluid (blue arrow). Dense aggregates of neutrophils fill alveoli. Photo and description courtesy of Dr Greg Stevenson. (Written communication with Greg Stevenson, DVM, PhD, lowa State University Veterinary Diagnostic Laboratory, July 5, 2019.)

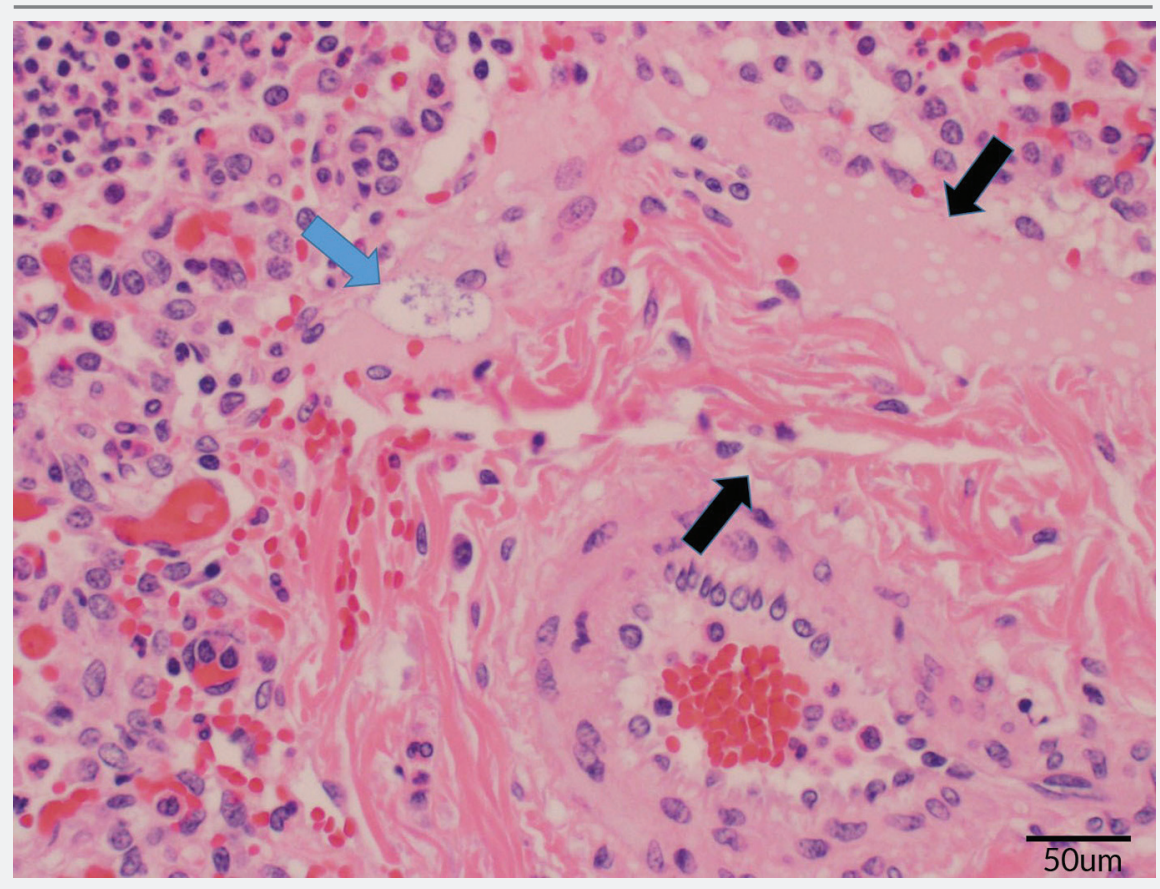

\section{Case 3}

The third case occurred the beginning of February 2020 at a 4-room, 2-barn site. Each of the 4 rooms had a capacity of 1250 pigs (5000 total capacity), and each room was naturally ventilated and partially slatted with solid block penning. The room placement populations were 1238 in room 1, 1238 in room 2, 1283 in room 3, and 1248 in room 4 for a total of 5007 pigs at the site. The flow of pigs at this farm were from a PRRS stable and PRRS MLV vaccinated flow. The older pigs at this site seemed normal and healthy but began dying suddenly the week prior to the veterinarian's visit. This flow of pigs previously had a clinical case of erysipelas, so tylvalosin tartrate (Aivlosin; Pharmgate) was administered via water assuming erysipelas was again the problem despite not seeing diamond skin lesions. The medication was ineffective, and younger pigs started dying suddenly just a few days prior to the veterinarian's visit. Some of the pens in the rooms had higher mortality than others, but the farm owner did not observe bloody discharge from the nose or mouth of dead pigs.

At the time of the veterinarian's visit, the pigs on the site ranged from 13 to 15 weeks of age and had been in the finisher for 4 to 6 weeks. Two pigs died during the veterinarian's walk-through and were chosen for necropsy and tissue collection. The lungs were adhered to the thoracic wall and were firm, heavy, and purple (Figure 1). There was also fibrin coating the heart. Like the first two cases, samples from the tonsil, lung, heart, multiple lymph nodes, liver, spleen, kidney, intestine, and colon were collected and sent to the ISU VDL where histopathology, culture, and PRRS, IAV, Mycoplasma hyorhinis, $M$ hyopneumoniae, and Glasserella parasuis (GPS) PCRs were completed. Microscopic lesions in the lungs included areas of parenchymal necrosis that were surrounded by fibrin, degenerated leukocytes, foci of hemorrhage, numerous degenerated neutrophils, macrophages, and occasional aggregates of streaming leukocytes (oat cells). A thick layer of fibrinous exudate also covered the pleura of the lung. In the heart, the pericardium was thickened and expanded by layers of granulation tissue (tissue formed in the process of healing). A fibrinous exudate covered the surface with scattered aggregates of lymphoplasmacytic infiltrates that multifocally extended into the myocardium. The mesothelial cells lining the pericardial surface were plumped and hypertrophied. 
Again, these lesions were consistent with an Actinobacillus infection. Actinobacillus suis was cultured from the lung and septicemic sites (liver and spleen). No other bacteria were cultured, and all PCRs were negative. The $A$ suis that was cultured was susceptible to ampicillin, ceftiofur, enrofloxacin, florfenicol, gentamicin, neomycin, penicillin, tiamulin, tilmicosin, and trimethoprim/ sulfamethoxazole.

Both tiamulin (Denagard) at the respiratory level and ceftiofur hydrochloride (Excenel RTU EZ) were recommended for this site to be used similarly to Cases 1 and 2 . The pigs remained at this site for 11 weeks after the veterinarian's visit. Thirty pigs from room 1 died in the remaining weeks before marketing for a total of 72 (5.82\%). Room 2 had 52 more pigs that died for a total mortality of 83 (6.70\%). Room 3 had the worst mortality with a total of 103 pigs (8.02\%), with 44 of those pigs dying in the last weeks. Room 4 lost 43 pigs in the remaining weeks for a total of 76 dead (6.09\%). The mean mortality across all 4 rooms was $6.67 \%$. Compared to historical annual mean mortality from 2018 (4.40\%) and 2019 (5.26\%), the mortality in this case was $2.27 \%$ and $1.41 \%$ more, respectively (Table 1). Again, finishing mortality is expected to increase by $1 \%$ to $2 \%$ with an A suis diagnosis. ${ }^{3}$

As was done in the other two cases described here, the healthy pigs were sold as quickly as possible to try to minimize mortality losses. The mean FILO age for this site during this case was 114 days. The 2018 historical mean FILO age was 129.87 days in 2018 and 126.16 days in 2019. These historical FILO age means were 15.87 and 12.16 days greater, respectively, than the mean FILO age for the pigs in this case (Table 1).

\section{Discussion}

Actinobacillus suis is a gram-negative, aerobic or facultative anaerobic, nonmotile, nicotramide adenine dinucleotide (NAD)-independent coccobacillus bacterium of the Pasteurellaceae family. ${ }^{1-3,6-10,12-14}$ It is a ubiquitous opportunistic pathogen and early colonizer of the tonsils and nasal cavity of healthy pigs. ${ }^{2,3,5,7-12,15-18}$ It can also be found in the vaginal mucosa of sows. , $^{2,3,8-10,12}$ Actinobacillus suis is believed to pass from sows to piglets. ${ }^{1,3,18}$ It is also transmitted by aerosol or nose-to-nose contact. $^{3,5}$ It is likely that the bacteria can invade through skin and mucous membrane abrasions as well., 9,10 However, this bacterium is susceptible to most disinfectants, can be killed if kept at $60^{\circ} \mathrm{C}$ for 15 minutes, and will die in clinical specimens after a few days., 9

Actinobacillus suis can be found in any age of pig but now most commonly occurs in early to middle finishing pigs. ${ }^{1,3,7-}$ 9,11,12,17 Stressful situations, such as transportation, concurrent diseases, crowding, poor ventilation, environmental extremes (excessive heat or frigid temperatures in the barns), among others, are risk factors associated with the incidence of clinical disease. ${ }^{1-3,5} \mathrm{De}$ creased immunity to $A$ suis due to being offspring of high-health animals, along with a concurrent influenza A(H1N2) virus infection, may have contributed to the $A$ suis diagnosis in Case 2. In Case 3 , the previous erysipelas diagnosis may have played a role in finding A suis. Other possible contributing risk factors for these cases are only speculations and therefore not mentioned here.

After the inciting stressful situation, $A$ suis invades through the upper respiratory tract and spreads systemically through the bloodstream. ${ }^{2}$ This can cause a variety of clinical signs, but often only sudden death is observed. $2,7,11$ Actinobacillus suis usually infects $10 \%$ or fewer of the pigs in the population, and it can be easily isolated from the pigs that die from the disease. ${ }^{1,4}$ Recently deceased pigs are therefore the best to necropsy especially in cases where sudden death is the only clinical sign like the cases described here. ${ }^{2,7,11}$ Petechial to ecchymotic hemorrhages can be observed in lung, kidney, liver, spleen, skin, and intestinal tissue samples. ${ }^{2,4,7,9,10}$ Actinobacillus suis can also produce fibrin similar to GPS and $S$ suis. $2,7,9,10,19,20$

There are three different forms of disease caused by A suis: an acute fulminant septicemic form, a respiratory form, and an acute septicemic form. 3,7,11 The acute fulminant septicemic form of $A$ suis usually occurs in younger growfinish pigs. $3,4,7,11,17$ Pigs with this form of the disease may have no other clinical signs other than death, and gross lesions are characterized by randomly scattered hemorrhages in multiple organs.4,7,11 Differentials for septicemia with similar lesions in younger grow-finish pigs include GPS, S suis, erysipelas, Escherichia coli, and Salmonella. . $2,4,7-10$ However, due to hemorrhages often seen in the lungs, APP should still be on the differential list. $^{9}$ This form of the disease was seen in Case 3.
The respiratory form mainly affects grow-finish pigs. ${ }^{3,4,7,11}$ Even in this form, sudden death is often the only clinical sign observed. ${ }^{3,7}$ Gross lesions include hemorrhages on multiple organs, but these are most often seen on the serosal surface of the lungs. ${ }^{3,7}$ The differentials should include APP because the gross and histologic lesions in the lungs of pigs with $A$ suis infections are indistinguishable from those of APP infections. ${ }^{1-3,6-11,18}$ Other differentials include GPS and erysipelas. ${ }^{9}$ This form of the disease was seen in Cases 1 and 2.

The acute septicemic form occurs in older growing pigs and adults. ${ }^{7}$ Skin lesions similar in appearance to erysipelas, abortions, metritis, fever, and anorexia can be observed in this form. 2,3,7,9,11,16 Again, hemorrhages in multiple organs and fibrin can be seen during necropsy. ${ }^{3,7}$ Differentials should include APP, GPS, and erysipelas. . $^{3,-9,11}$

Pulmonary lesions caused by APP and A suis are often indistinguishable. ${ }^{9}$ Therefore, both bacteria should be differentials when the clinical signs and gross lesions described here are seen. The primary reason the lesions of these two diseases are almost identical is due to the production of toxins. ${ }^{9}$ The toxins produced by $A$ suis are ApxI and ApxII, both of which are also produced by APP. 2,7,9,11,12,15-18 However, A suis is less virulent than APP because $A$ suis is believed to produce fewer of these toxins than APP. ${ }^{2,4,7}$

Bacterial culture is the gold-standard test for A suis diagnosis. ${ }^{2,6-10}$ Actinobacillus suis grows well on blood agar plates, and it can be found in both the lungs and systemic sites. ${ }^{2,3,6,9,10,18}$ Actinobacillus suis was cultured from the lungs in Cases 1 and 2 and from the lung, spleen, and liver in Case 3.

An $A$ suis PCR was not done for these three cases. While no PCR test has been validated for A suis, some laboratories may offer this test. An $A$ suis enzymelinked immunosorbent assay has also not been validated. However, an experimental antibody test for $A$ suis is being used at the University of Montreal. ${ }^{16}$ While still experimental, Lapointe et al ${ }^{16}$ points out that this test may be useful in making decisions regarding the use of autogenous vaccines but should not be used diagnostically.

To decrease mortality in suspected A suis outbreaks, it is necessary to start treatment as soon as possible, prior to receiving sample results back from the 
lab. The inability to clinically distinguish between APP and $A$ suis guided the veterinarian's choice of antibiotics used for the 3 cases. ${ }^{1-3,6-11}$ For $A$ suis, there are multiple choices for water-administered antibiotics including tiamulin, chlortetracycline, sulfadimethaxine, oxytetracycline, gentamicin, neomicin, clindamycin, and penicillin., ${ }^{1,7}$ These can be used with varying efficacy according to laboratory minimum inhibitory concentrations, though none are labeled for A suis. ${ }^{1}$ While both chlortetracycline and tiamulin are labeled for APP, reports of chlortetracycline resistance may make tiamulin the only water-administered antibiotic on this list that is effective for APP. ${ }^{7}$ Ceftiofur and gentamicin are injectable antibiotics that are effective against both APP and A suis. ${ }^{7}$ However, of the two, only ceftiofur is labeled for APP and neither are labeled for A suis.

Currently, there are no effective commercial vaccines available for $A$ suis. The production of a vaccine could help lower the chances of devasting disease by increasing humoral immunity and stabilizing antibodies. ${ }^{16}$ Lapointe et $\mathrm{l}^{16}$ described an autogenous vaccine that was produced using two field isolates of the bacteria. Protocols for autogenous vaccine use varies depending on the age of disease onset. ${ }^{16}$ Vaccination of sows is warranted for repeated disease seen in piglets, but pigs should be vaccinated if the disease repeatedly occurs in finishing. ${ }^{16}$ The author did not pursue the production of an autogenous vaccine due to the sporadic nature of disease seen across multiple flows.

\section{Implications}

Under the conditions of this case study:

- Bacterial culture was a valuable diagnostic test for cases of $A$ suis infection.

- Treatment that targets both APP and $A$ suis infections should be considered.

\section{Acknowledgments}

The author would like to thank Dr Greg Stevenson at the ISU VDL for sending the histological pictures and descriptions and Dr Eric Burrough at the ISU VLD for his technical assistance. The expertise from Drs Karen Harmon and David Baum at the ISU VDL on PCR and serologic testing was also appreciated.

\section{Conflict of interest}

None reported.

\section{Disclaimer}

Scientific manuscripts published in the Journal of Swine Health and Production are peer reviewed. However, information on medications, feed, and management techniques may be specific to the research or commercial situation presented in the manuscript. It is the responsibility of the reader to use information responsibly and in accordance with the rules and regulations governing research or the practice of veterinary medicine in their country or region.

\section{References}

*1. Hanthorn C, Schwartz KJ. Actinobacillus suis: does data support what we "know"? Proc of the $39^{\text {th }}$ AASV Annual Meeting. American Association of Swine Veterinarians; 2008:303-312.

2. Neumann EJ, Ramirez A, Schwartz KJ. Actinobacillus suis. In: Neumann EJ, Ramirez A, Schwartz KJ, eds. Swine Disease Manual. $5^{\text {th }}$ ed. American Association of Swine Veterinarians; 2020:12-13.

*3. Dufresne, L. Personal experience with Actinobacillus suis. Proc Allen D Leman Swine Conference. University of Minnesota; 2006:87-98.

4. MacInnes JI, Desrosiers R. Agents of the "Suis-ide Diseases" of Swine: Actinobacillus suis, Haemophilus parasuis, and Streptococcus suis. Can J Vet Res. 1999;63:83-89.

*5. Madson D. Actinobacillus suis: a description of one integrated system's field challenges. Proc Allen D Leman Swine Conference. University of Minnesota; 2006:90-92.

6. Yaeger M. An outbreak of Actinobacillus suis septicemia in grow/finish pigs. J Vet Diagn Invest. 1996;8:381-383.

7. Gottschalk, M. Actinobacillosis. In: Zimmerman JJ, Karriker LA, Ramirez A, Schwartz KJ, Stevenson GW, eds. Diseases of Swine. $10^{\text {th }}$ ed. Wiley-Blackwell; 2012:653-669.

8. Kariyawasam S, Strait E, Jordan D, Kroll J. Development of a real-time polymerase chain reaction assay for detection of Actinobacillus suis in porcine lung. $J$ Vet Diagn Invest. 2011;23(5):885-889.

*9. Oliveira S. Update on Actinobacillus suis diagnosis, epidemiology, and control: On the path from good to great. Proc of the $38^{\text {th }}$ AASV Annual Meeting. American Association of Swine Veterinarians. 2007:371-376.
*10. Oliveira S. Actinobacillus suis: an update on genotyping and antibiotic susceptibility. Proc Allen D Leman Swine Conference. University of Minnesota. 2006:93-95.

*11. Gunn-McQuillan H, MacInnes J. Prevalence and significance of Actinobacillus pleuropneumoniae and Actinobacillus suis in Ontario. Proc of the $36^{\text {th }} \mathrm{AASV}$ Annual Meeting. American Association of Swine Veterinarians. 2005:55-62.

12. Ganeshapillai J, Boncheff AG, Slavic D, MacInnes J, Monterio MA. The lipopolysaccharide core of Actinobacillus suis and its relationship to those of Actinobacillus pleuropneumoniae. Biochem Cell Biol. 2011;89:351-358.

*13. Actinobacillus suis. Iowa State University Swine Disease Manual. Accessed January 20, 2021. https://vetmed.iastate. edu/vdpam/FSVD/swine/index-diseases/ actinobacillus-suis

14. Frey J. The role of RTX toxins in host specificity of animal pathogenic Pasteurellaceae. Vet Microbiol. 2011;153:51-58.

15. Kamp EM, Vermeulen TMM, Smits MA, Haagsma J. Production of Apx toxins by field strains of Actinobacillus pleuropneumoniae and Actinobacillus suis. Infect Immun. 1994;62(9):4063-4065.

16. Lapointe L, D'Allaire S, Lacouture S, Gottschalk M. Serologic profile of a cohort of pigs and antibody response to an autogenous vaccine for Actinobacillus suis. Vet Res. 2001;32(2):175-183.

17. MacInnes JI, Gottschalk M, Lone AG, Metcalf DS, Ojha S, Rosendal T, Watson SB, Friendship RM. Prevalence of Actinobacillus pleuropneumoniae, Actinobacillus suis, Haemophilus parasuis, Pasteurella multocida, and Streptococcus suis in representative Ontario swine herds. Can J Vet Res. 2008;72:242-248.

*18. Oliveira S. Diagnostic update: Haemophilus parasuis, Actinobacillus suis, and Actinobacillus pleuropneumoniae. Proc of the $40^{\text {th }}$ AASV Annual Meeting. American Association of Swine Veterinarians. 2009:449-452.

19. Neumann EJ, Ramirez A, Schwartz KJ. Streptococcus suis. In: Neumann EJ, Ramirez A, Schwartz KJ, eds. Swine Disease Manual. $5^{\text {th }}$ ed. American Association of Swine Veterinarians; 2020:50-52.

20. Gottschalk, M. Streptococcosis. In: Zimmerman JJ, Karriker LA, Ramirez A, Schwartz KJ, Stevenson GW, eds. Diseases of Swine. $10^{\text {th }}$ ed. Wiley-Blackwell; 2012:841-855.

* Non-refereed references. 